\title{
Neuroform-EZ Stent Modification for Facilitated Delivery during Intracranial Aneurysm Embosurgery
}

\author{
Walter S. Lesley, MD, CPE, FACR ${ }^{1,2}$, Delip V. Patel, MD, MBA'
}

The Neuroform EZ Stent System is a fourth generation intracranial aneurysm stent that utilizes an integrated navigation guidewire. While designed to facilitate stent delivery, the guidewire can rarely impede proper positioning of the stent. In this technical case report, severing the guidewire from the stent delivery system with surgical scissors was required for successful stent implantation.

Key Words : Stent; Neuroform; Aneurysm

The Neuroform EZ Stent System (Boston Scientific Corporation, Fremont, CA, USA) is a fourth generation intracranial aneurysm stent system approved for use in the US by the Food and Drug Administration to treat wide-neck aneurysms in conjunction with endovascular coiling [1]. Predicated on the technology of early generation stent systems, there have been structural and mechanical modifications to the Neuroform EZ Stent System (NF-EZ) to facilitate positioning, deployment, and vascular accessibility. The NF-EZ Directions for Use (DFU) outlines a standard technical approach and discourages physical tampering of the stent system. In this technical note, the standard stent deployment was

\footnotetext{
'Departments of Radiology, ${ }^{2}$ Surgery and Neurosurgery, Division of Neuroradiology, Section of Neurolnterventional Surgery, Texas A\&M University Health Sciences Center College of Medicine/Scott \& White HealthCare, TX, USA

Received May 18, 2013; accepted after revision August 13, 2013.

Correspondence to: Walter S. Lesley, MD, CPE, FACR, 2401

South 31rst St, Suite 2X Temple, TX, 76508, USA

Tel. 254.724.2522 Fax. 254.724.0502

E-mail: wlesley@sw.org

This is an Open Access article distributed under the terms of the Creative Commons Attribution Non-Commercial License (http://creativecommons.org/licenses/by-nc/3.0) which permits unrestricted non-commercial use, distribution, and reproduction in any medium, provided the original work is properly cited.
}

impeded as a result of the newly integrated preshaped distal guidewire. To overcome this, the NF-EZ guidewire was removed using surgical scissors. Successful stent implantation and aneurysm coiling were consequently achieved.

\section{TECHNICAL CASE REPORT}

An adult male was discovered unconscious at home and a CT head demonstrated diffuse subarachnoid hemorrhage, mild ventricular hemorrhage and hydrocephalus. On subsequent CT angiogram of head, a moderately wide neck $3 \mathrm{~mm}$ left anterior communicating artery (ACom) aneurysm was identified. The patient was taken to the neurointerventional suite, where the small ACom aneurysm was successfully coiled (Axium, Covidien Corporation, Plymouth, MN, USA). On 6-month follow-up MR angiogram of the head, a small recurrent aneurysm was discovered, so a stage-II embosurgery was scheduled.

The patient was pre-operatively medicated with aspirin and clopidogrel. Under general anesthesia, a 6French right femoral artery sheath was placed. Intravenous heparin and $1 \mathrm{~g}$ of cefazolin were administered. A 6-French guidecatheter was placed in the left ICA. Using multiple microcatheters and microwires in 
varying combinations, the intracranial circulation was explored. The recurrent aneurysm was noted to be small, measuring about $2 \mathrm{~mm}$, and which demonstrated a wide base that incorporated portions of the left ACom and the left A2 (Fig. 1A). Given this configuration plus the associated tortuous vascularity, stent-assisted coiling was elected. A Marksman catheter (Covidien Corporation) was primarily advanced over numerous microwires in an unsuccessful attempt to gain access in the left A2 distal to the aneurysm. As a result, exchange wire technique was used: An SL-10 microcatheter (Boston Scientific Corporation, Fremont, CA, USA), was successfully advanced over exchange length Synchro2 microwire (Boston Scientific Corporation). With the wire at the left $\mathrm{A} 2-3$ junction, the SL-10 microcatheter was withdrawn. Over this exchange wire, the Marksman catheter was advanced as far as possible into the left A2, that is, approximately $1 \mathrm{~cm}$ beyond the aneurysm (Fig. 1B). Advancing this catheter any further was prevented by a combination of the general vascular tortuosity and the relatively small caliber of the A2. A $2.5 \times 15 \mathrm{~mm} \mathrm{NF-EZ} \mathrm{stent} \mathrm{was}$ brought to the field, prepped, and advanced through the Marksman catheter. Though the NF-EZ stent system advanced easily, the Marksman catheter slightly retreated with its tip adjacent to the most proximal A3 subfrontal branch. The NF-EZ guidewire readily entered this small vessel instead of continuing around into the distal A2. An attempt to advance the stent with the wire in this small branch was met with additional resistance and threatened further retreat of the Marksman catheter. Because of the added length of the NF-EZ guidewire, the stent apparatus could not be positioned completely across the aneurysm orifice, and
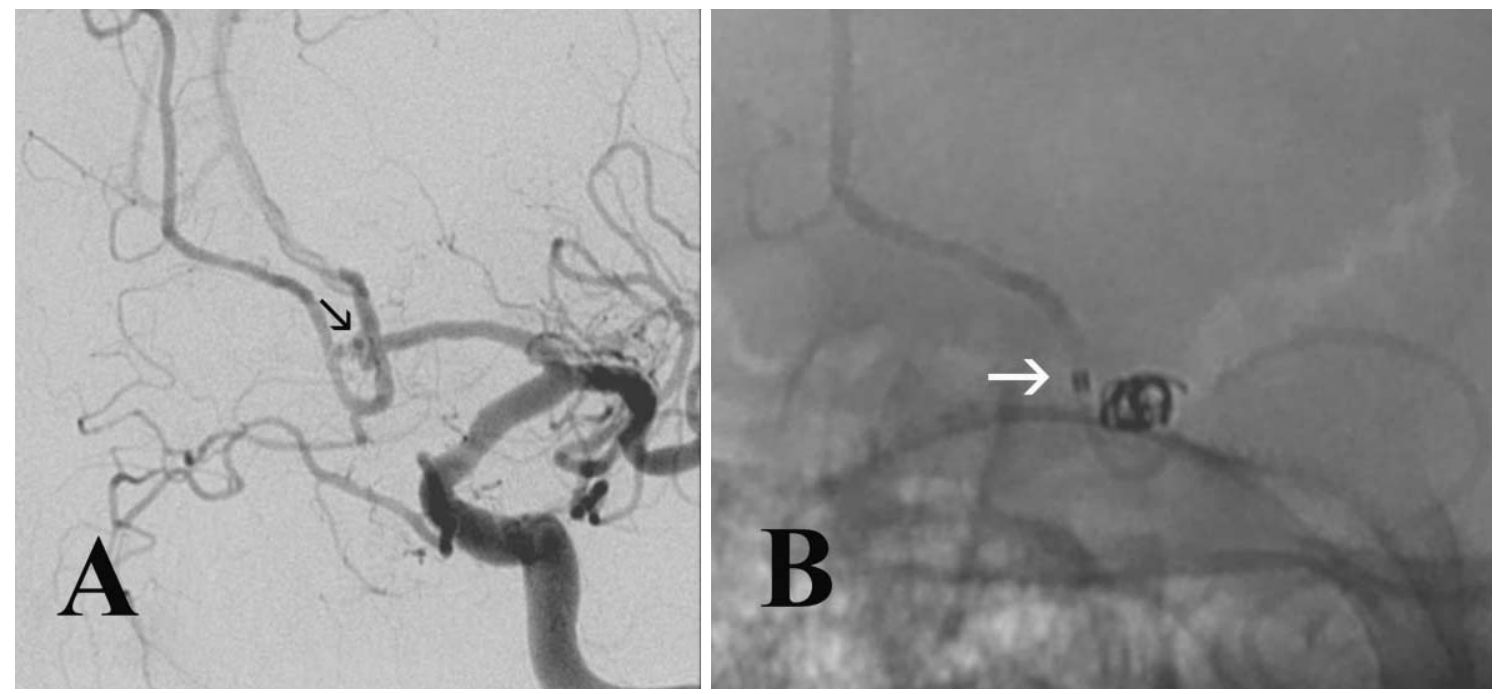

Fig. 1. Stage-2 intraoperative angiogram demonstrates the recurrent, coiled ACom aneurysm (A, arrow). The most distal position of Marksman catheter (B, arrow) is shown prior to its slight retreat during the initial attempt at introducing the unmodified NF-EZ. Note that this microcatheter has a similar diameter to the left $\mathrm{A} 2$.
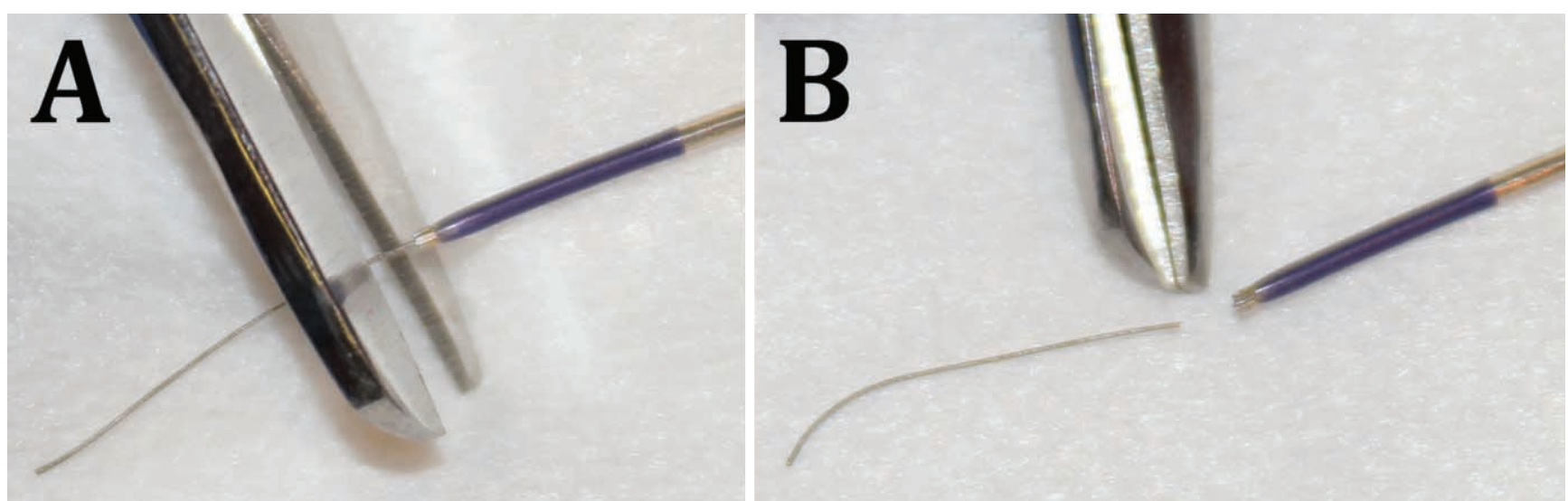

Fig. 2. The appearance of the NF-EZ stent guidewire and distal bumper are shown before (A) and after modification using surgical scissors. 


\section{Neuroform-EZ Stent Modification for Facilitated Delivery}

so it was withdrawn. The stent apparatus was examined and found to be free of defect or otherwise adversely affected by the attempt to place it. But to gain sufficient intracranial purchase of the stent, the guidewire was snipped off flush using surgical scissors at the junction with the distal stent bumper (Fig. 2). The stent was readvanced into the Marksman catheter and easily placed across the aneurysm neck. With the distal guidewire now removed, care was taken to ensure that forward motion of the stent delivery system did not advance beyond the tip of the Marksman catheter. With the stent positioned across the aneurysm, the Marksman catheter was manually withdrawn, unsheathing the stent in an acceptable position-- completely crossing the aneurysm orifice, extending from the left A2 into the left A1 (Fig. 3). The stent delivery system was then removed without disturbing the stent. Aneurysm coiling proceeded uneventfully at that point (Deltapaq and Deltaplush, Codman \& Shurtleff Inc, Raynham, MA, USA). Final angiography demonstrated an adequately packed aneurysm with coils completely secured in the aneurysm by the stent (Fig. 4). The patient did well post-operatively, and remains neurologically intact, functionally independent and fully employed (most recent follow-up at 15 months). Because the above NF-EZ modification represented a device protocol deviation, it was reported to both the local IRB and the device's sponsor in a timely fashion.

\section{DISCUSSION}

The NF-EZ is the latest offering from the Neuroform stent family. Like its predecessors, the NF-EZ stent

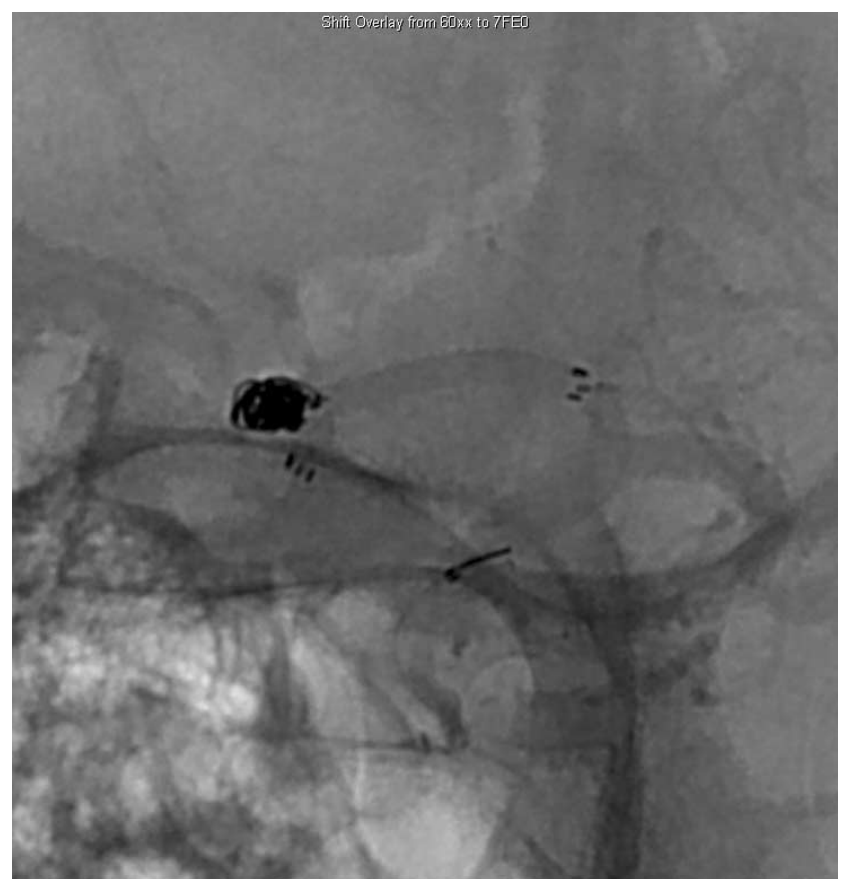

Fig. 4. Native angiogram of stent implant and final coil placement demonstrates complete covering of the aneurysm by the stent and a secured aneurysm.

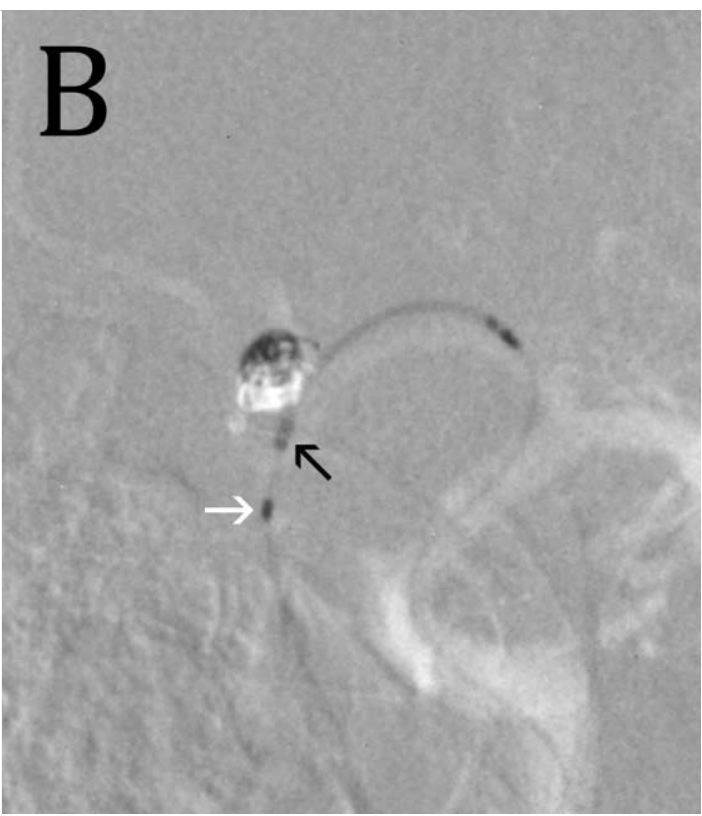

Fig. 3. A. lateral oblique; B. anterior oblique angiograms.

With the NF-EZ guidewire removed, the distal stent bumper marker (white arrow) has been advanced nearly to the tip of the Marksman catheter (black arrow), which has now engaged the left A3 subfrontal branch (A). The distal stent bumper (white arrow) remains adequately beyond the aneurysm orifice during manual withdrawal of the catheter (B). 
renders mechanical and hemodynamic benefits for aneurysm repair by preventing and repairing coil protrusion into the parent artery, reducing intraaneurysmal flow, and promoting aneurysm occlusion [2-4]. The NF-EZ confers several advantages over the existing third generation Neuroform Stent Delivery System. Some of the technical difficulties described with the third generation version included the en bloc advancement or transfer to a pre-positioned alternate microcatheter, which can also be time consuming and technically challenging for a single operator.

Complications can arise in conjunction with stent implantation including stent migration, maldeployment and stent herniation into the aneurysm $[5,6]$. However, the NF-EZ allows the sole surgeon to more easily place the stent without the challenges of handling a long stent transfer system. Also, the addition of distal and proximal radioopaque positioning bumpers and a preshaped distal tip have, overall, facilitated precise positioning prior to, and during deployment.

In regards to this unique device modification, the standard stent pre-deployment technique failed because of mechanical impendence from the preshaped distal tip entering a small vessel branch. Friction generated from the patient's intra- and extracranial vascular tortuosity further limited the amount of forward pressure that could be placed upon the delivery and guide catheters without causing these catheters to retreat. A larger caliber or stiffer guide catheter might have obviated the need to modify the stent. Also, a third, intermediate catheter (e.g., Navien, Covidien Corporation) would potentially have offered a more robust tri-axial support system.

The Wingspan stent (Stryker, Kalamazoo, MI, USA) was considered as a back-up option if the rigid nosecone of this stent was likewise severed as previously reported by Lesley et al [7]. But owing to the Wingspan's stent inherently greater stiffness compared to the Neuroform stent, the off-label choice of this stent was avoided. Other intracranial stents (Solitiare, Covidien Corporation; and, Enterprise, DePuY/ Codman \& Shurtleff, Inc, Raynham, MA, USA) as well as the Navien catheter were not stocked in-house at the time of the procedure and were therefore not considered in this case.

Any perceived benefit must be weighed against the potential for patient harm when modifying any surgical device. Before introducing the altered stent, the guide wire and distal stent bumper were meticulously scrutinized. The surgical scissors had cleanly amputated the wire from the stent, leaving no wire remnant or jagged edge. The modified stent advanced readily to the tip of the microcatheter with no tactile sensation of resistance and no fluoroscopic evidence of unexpected device function. Because of the blunt nature of the distal stent bumper, extreme care was used in the absence of the stent guide wire to ensure that the bumper did not advance unprotected within the vessel during the microcatheter unsheathing of the stent.

The NF-EZ is overall, an improved iteration compared to its predecessors. However, the preshaped distal guidewire can, on occasion, impede sufficient distal placement of the stent. Snipping off the distal guidewire may be safely performed to overcome this obstacle.

\section{ACKNOWLEGEMENT}

The authors have no personal or institutional financial interest in drugs, materials, or devices described in this publication.

\section{References}

1. Neuroform EZ Stent System. Directions for use. Fremont (CA): Boston Corporation, 2012.

2. Gu DQ, Zhang X, Luo B, Long XA, Duan CZ. The effect of Neuroform stent-assisted coil embolization of wide-necked intracranial aneurysms and clinical factors on progressive aneurysm occlusion on angiographic follow-up. J Clin Neurosci 2013;20:244-247

3. Santillan A, Greenberg E, Patsalides A, Salvaggio K, Riina HA, Gobin YP. Long-term clinical and angiographic results of Neuroform stent-assisted coil embolization in wide-necked intracranial aneurysms. Neurosurgery 2012;70:1232-1237

4. Kim YJ. Early experiences of neuroform stent-assisted coiling in ruptured intracranial aneurysms. Interv Neuroradiol 2007;13:3144

5. Thomas JA, Watson VE, McGrail KM. Surgical management of a paraclinoid aneurysm containing a displaced neuroform microstent: technical case report. Neurosurgery 2008;63:E817

6. Yahia AM, Gordon V, Whapham J, Malek A, Steel J, Fessler RD. Complications of neuroform stent in endovascular treatment of intracranial aneurysms. Neurocrit Care 2008;8:19-30

7. Lesley WS, Rangaswamy R. Catheter exchange technique for the Wingspan stent: technical note. J Neurointerv Surg 2009;1:168-70 